\section{Cystic intra-abdominal masses in children}

\author{
Luisa Ferrero, Riccardo Guanà, \\ Giulia Carbonaro,
} Maria Grazia Cortese, Luca Lonati, Elisabetta Teruzzi, Jurgen Schleef

Division of Pediatric General, Thoracic and Minimally Invasive Surgery, AOU Città della Salute e della Scienza, Regina Margherita Children's Hospital, Torino, Italy

\section{Abstract}

Benign intra-abdominal cystic masses in infancy are fairly uncommon and their etiopathogenesis, histology and clinical presentation differ significantly. Our aim is to report our experience in their treatment in order to discuss the best diagnostic and treatment modality. The medical records of 5 children $(2 \mathrm{M}, 3 \mathrm{~F})$ with cystic intraabdominal masses referred to our hospital between November 2012 and September 2016, were retrospectively reviewed. All patients underwent open surgery and subsequent histopathologic analysis. Different clinical presentations, localizations of the masses, diagnostic tools, surgical approaches, histological examinations and outcomes were reviewed. Patients mean age was 5.4 years (range: 8 months-9 years). Two patients presented recurrent abdominal pain and abdominal distension; 1 patient had a palpable mass discovered incidentally and 2 complained acute abdominal pain. Routine laboratory tests, tumor markers and abdominal ultrasound were immediately done in all patients. Three patients underwent MRI and 1 abdominal CT. At laparotomy 2 hepatic cysts, 2 mesenteric cyst and 1 retroperitoneal cyst were discovered. Histology reports described: 1 hepatobiliary cystadenoma, 1 benign hepatic hamartoma and 3 cystic lymphangiomas (1 retroperitoneal and 2 mesenteric). There were no major postoperative complications, deaths, or recurrences in our series (follow-up 3-24 months). Despite the rarity of these lesions, benign cystic abdominal masses in children are not so uncommon and should be considered as causes of acute abdominal pain. The differential diagnosis is not always possible preoperatively. In our series, radical excision of the lesions was possible in all cases, allowing reliable histological results and avoiding recurrences.

\section{Introduction}

Benign intra-abdominal cystic masses in infancy and childhood are uncommon and their etiopathogenesis, histology, localization and clinical presentation differ significantly; this could generate diagnostic dilemmas and consequent therapeutic delay.

As cystic mass we considered a newlyformed closed cavity with liquid or semisolid contents and an epithelial lining, while fluid-filled masses that lack an epithelial lining were intended as cyst-like. ${ }^{1}$

Here we describe 5 patients affected by cystic intra-abdominal masses surgically treated, focusing on diagnostic tools and trying to find similarities between them, and we review similar lesions in children, in the recent literature.

Our hospital is a tertiary-referral Center that treats children from 0 to 13 year old; in the study period we have operated on the following solid abdominal tumors: 15 neuroblastomas, 12 nephroblastomas (Wilms tumor), 3 ovarian cystoadenomas, 5 ovarian teratomas, 1 colonic tumor (Gastrointestinal Autonomic Nerve Tumor, GANT), 1 rahbdomyosarcoma and 5 Hodgkin's lymphomas.

\section{Methods}

The medical records of 5 children $(2 \mathrm{M}$, $3 \mathrm{~F})$ with cystic intra-abdominal masses referred to our hospital between November 2012 and September 2016, were retrospectively reviewed.

All patients underwent open surgery and subsequent histopathologic analysis.

Different clinical presentations, localizations of the masses, diagnostic tools, surgical approaches, histological examinations and outcomes were reviewed.

Informed consent was obtained from children's parents to manage patients clinical data.

\section{Case Report \#1}

A previously healthy 9-year-old girl complained of chronic abdominal pain since one year.

She was finally admitted to our Emergency Department (ED) in November 2012, because of acute abdominal pain, diarrhea and fever. On physical examination, the abdomen was tense but tender and a large, hard in consistency, fixed palpable mass was detected in the right hypochondrium. Liver function tests revealed augmented values of GOT (164 U/L), GPT (230 $\mathrm{U} / \mathrm{L}$ ) and on the ultrasound examination a
Correspondence: Riccardo Guanà, Division of Pediatric General, Thoracic and Minimally Invasive Surgery, Regina Margherita Children's Hospital, Piazza Polonia 94, 10126, Turin, Italy. Tel.: +39.011.3131792 - Fax: +39.011.3135060

E-mail: riccardoguan@gmail.com

Key words: Cystic abdominal masses; hepatic hamartoma; hepatobiliary cystadenoma; lymphangioma.

Contributions: the authors contributed equally.

Conflict of interest: the authors declare no potential conflict of interest.

Received for publication: 28 June 2017.

Revision received: 8 August 2017.

Accepted for publication: 11 August 2017.

This work is licensed under a Creative Commons Attribution NonCommercial 4.0 License (CC BY-NC 4.0).

(C) Copyright L. Ferrero et al., 2017

Licensee PAGEPress, Italy

Pediatric Reports 2017; 9:7284

doi:10.4081/pr.2017.7284

multiloculated cystic mass (maximum diameter $15 \mathrm{~cm}$ ) was noted, arising from the liver right lobe (Figure 1A).

Abdominal MRI with intravenous contrast, revealed a $15 \times 9.5 \times 9 \mathrm{~cm}$ multiloculated, thinly septated cystic mass that occupied the majority of the right upper abdomen (Figure 1B,C); a clear plane between the mass and the liver or the colon could not be precisely identified, making the primary origin unclear.

The patient underwent an exploratory laparotomy through a bi-sided subcostal incision and the large cystic mass was easily identified, arising from the right lobe of the liver. The mass was exophytic, well encapsulated, and essentially occupied the $\mathrm{V}$ and VI segments of the liver, without invading any other structure (Figure 1D).

The mass was completely and radically excised, intact, with an atypical s5 and s6 resection.

Gross pathologic examination described an intrahepatic multiloculated cystic mass with a thick capsule and smooth inner lining that contained gelatinous bile stained fluid (Figure 1E). Microscopically, the cystic lesion was lined by biliary type epithelium.

The surgical margins were negative for residual tumor and the histopathologic diagnosis was hepatobiliary cystadenoma.

The patient's post-operative course was uneventful and her 2-year follow-up was negative for recurrences. 


\section{Case Report \#2}

A 1-year-old boy presented to our ED in November 2012 with chronic constipation and sudden abdominal distension.

On physical examination, a palpable mass was detected in the right hypochondrium. Liver function tests reveled augmented values of GOT (376 U/L) and GPT (230 $\mathrm{U} / \mathrm{L}$ ) while tumor markers were negative.

Abdominal ultrasonography and MRI revealed a $9.2 \times 8.3 \times 9.2 \mathrm{~cm}$ multiloculated well-defined, firm mass arising from the right lobe of the liver and that displaced the hepatic parenchyma (cranially) and the bowel (Figure 2A).

The patient underwent laparotomy by a bi-subcostal incision. A mass arising from the $\mathrm{V}$ and VI segments of the liver was identified (Figure 2B) and was totally resected by enucleation.

A histological diagnosis of benign mesenchymal cystic liver hamartoma was made. The patient recovered well and was discharged from the hospital on day five; the two-year follow-up was negative for recurrences.

\section{Case Report \#3}

A 5-year-old girl was evaluated by the general practitioner (GP) in January 2013 for moderate abdominal pain which had been lasting for 3 days.

On abdominal ultrasound, a large fluid collection containing internal debris in the inferior abdominal quadrants was noted, and a diagnosis of ovarian cyst was made.

She was referred to our ED where the physical examination was negative for palpable masses and the tumor markers revealed a mild augmentation of CA-125.

Abdominal CT-scan with intravenous contrast confirmed the presence of a $12 \times$ $15 \times 5 \mathrm{~cm}$ well-defined, cystic mass localized in the sub-hepatic quadrant and expanding down to the pelvis (Figure 3A).

The girl was submitted to laparotomy by a sovraumbilical transversal incision and a large cystic lesion (500 g of weight) with lymphatic content, arising from the jejunoileal mesentery, was identified.

Total excision of the lesion required a segmental bowel resection (Figure 3B).

The histopathologic examination of the cystic mass revealed a multiloculated cyst with a columnar mesothelial lining and no cellular atypia, compatible with a mesenteric cystic lymphangioma.

The postoperative course was uneventful with no recurrence of the mass after an 18-month follow-up.

\section{Case Report \#4}

A previously healthy 7-year-old boy was admitted to our ED in March 2013, for a periumbilical, palpable mass incidentally detected by the GP.

Abdominal ultrasonography and MRI revealed two contiguous, non-enhancing cystic lesions $(5.6 \times 4.4 \times 5.4 \mathrm{~cm}$ and $6.7 \times$ $4.2 \times 7.6 \mathrm{~cm}$ respectively) in the right midabdomen, caudally to the right kidney and above the vertebral plan. The mass displaced the bowel to the left and anteriorly (Figure 4).

An explorative laparoscopy was attempted with a $10 \mathrm{~mm}$ trocar inserted in the umbilicus with an open technique. A large bi-lobated cystic mass occupied the right quadrant of the abdomen.

After placing 1 additional operative 5 $\mathrm{mm}$ trocar, the cyst was mobilized and was noted to arise from the retroperitoneum. Conversion to open surgery was the next step. A median sub-umbilical incision was made. Total excision was easily performed with gentle detachment of the lesion from the duodenum, the right ureter and the inferior vena cava.

Histological diagnosis of retroperitoneal cystic lymphangiomas was made.
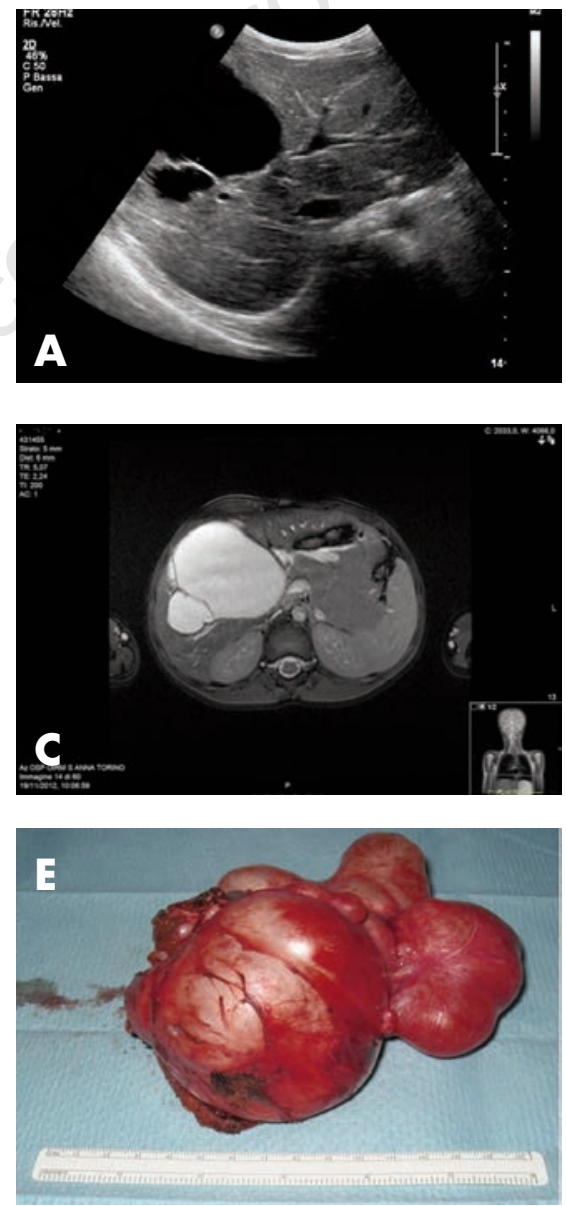

The postoperative course was uneventful with no recurrence of the mass after 18month follow-up.

\section{Case Report \#5}

A 2-year-old girl was hospitalized previously in another structure and than transferred to our Unit for acute abdominal pain, in September 2016.

At the abdominal ultrasound we noted a cystic abdominal mass in the left hypochondrium, $10 \mathrm{~cm}$ in diameter, septated; the abdominal CT-scan evidenced a hypodense mass, $11 \mathrm{~cm}$ in diameter, arising from the small gastric curvature (Figure 5).

At laparotomy, through a transverse supraumbilical left abdominal incision, we evidenced a large multicystic mass arising from the little omentum and contracting strong adhesions with the gastric wall. We performed an en bloc resection with a portion of the gastric wall. A frozen section biopsy excluded the malignant nature of the lesion, which had the macroscopic aspect of an abdominal lymphangioma.

Postoperative period was uneventful and histological diagnosis was mesenteric lymphangioma.
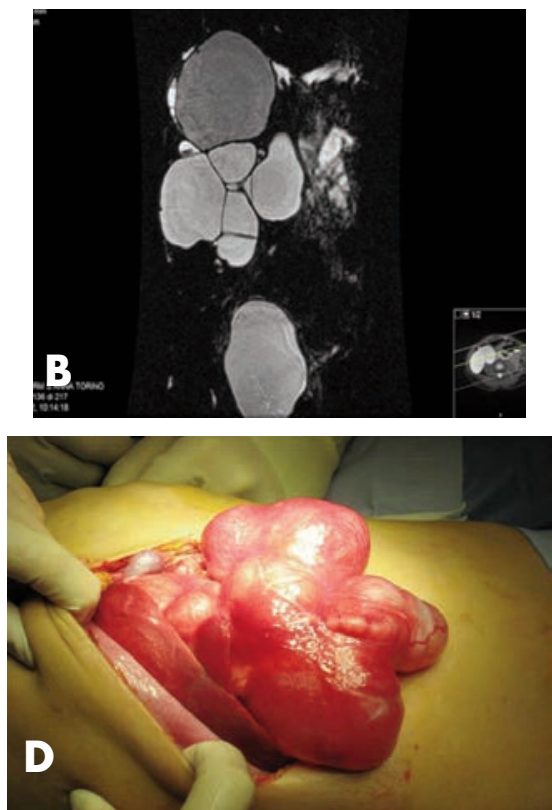

Figure 1. A) Ultrasound scan of the multiloculated cystic mass of the hepatobiliary cystadenoma. B) Computed tomography scan coronal view of the multiloculated, thinly septated cystic mass in the right upper abdomen described in the first case. C) Computed tomography scan transversal view of the mass. D) Intraoperative findings. E) Excised mass after atypical hepatic resection. 


\section{Discussion}

Intra-abdominal cystic masses are traditionally classified by location and histology. ${ }^{2}$ It is often difficult to characterize them pre- and intra-operatively, mostly when they are very large and fill the entire abdomen.

They sometimes distort near organ anatomy and their site of origin can be difficult to ascertain.

An intra-abdominal cystic mass can originate not only from solid organs such as the liver, the spleen, and the pancreas but also from the retroperitoneum, the omentum and the mesentery.

Affected patients present with various symptoms and physical findings, depending on size, localization, and mass effect on the adjacent abdominal structures. In most cases the cystic abdominal masses can be incidental findings.

The symptoms can be abdominal pain, early satiety, bowel obstruction or fever; the physical examination shows abdominal distention or a palpable mass.

Abdominal ultrasound is the imaging of
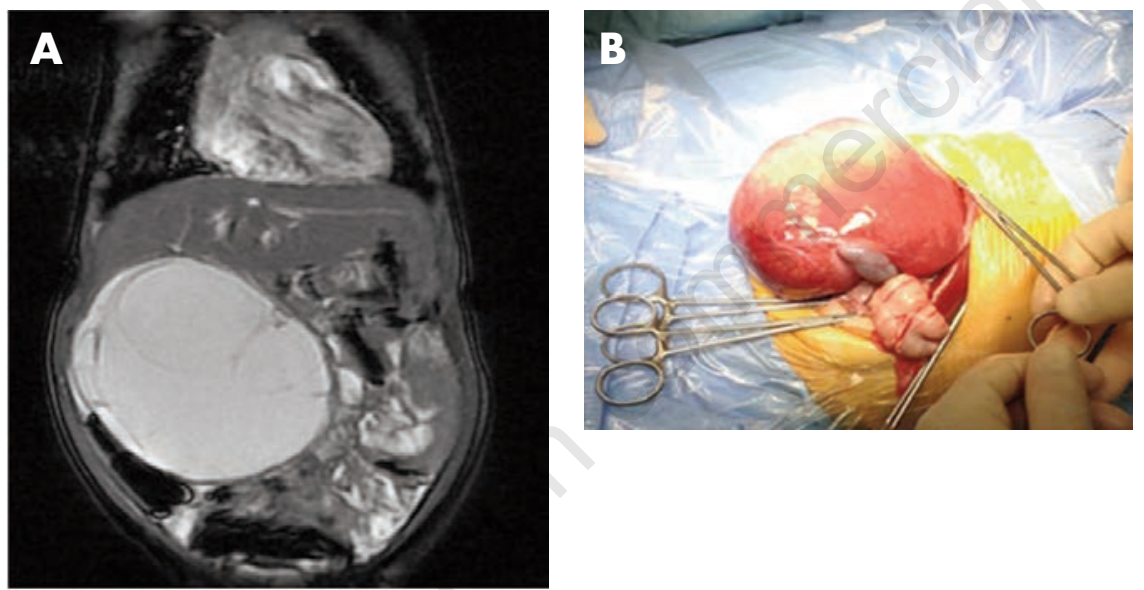

Figure 2. A) Magnetic resonance imaging coronal view of the multiloculated cystic mass in the right upper abdomen (benign hepatic mesenchymal cystic liver hamartoma). B) Intraoperative findings.
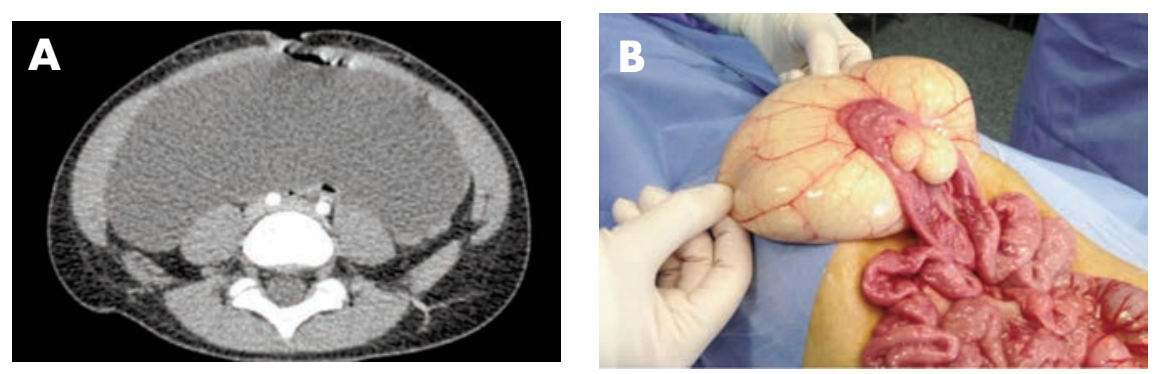

Figure 3. A) Computed tomography scan transversal view of the mass (mesenteric cystic lymphangioma). B) Operative findings demonstrating multiple cysts involving the jejunoileal mesentery. Total excision of the lesion required a segmental bowel resection. tadenomas that may occasionally occur in male patients as well. 6

Since their initial description, cystadenomas have been increasingly reported in literature.

The etiology of hepatobiliary cystadenoma is unclear: the prevailing theories attribute the lesion to congenital anomalies of the biliary tract. Due to the paucity of cases in the pediatric population, this lesion is rarely diagnosed preoperatively. The most useful non-invasive radiographic studies in evaluating this hepatic lesion are the abdominal ultrasound and CT-scan.

Various treatment modalities have been used to manage hepatobiliary cystadenoma, including simple aspiration, drainage, marsupialization, enucleation, and complex excision.

Because, at present, only ten cases have been described in the pediatric population, there is no coded treatment algorithm for hepatobiliary cystadenoma in children.

Based on data extrapolated from the adult literature, it is accepted that the most appropriate management is complete tumor resection. In fact, incompletely excised lesions may result in tumor recurrence. 6

Furthermore, hepatobiliary cystadenomas that otherwise contain benign-appearing epithelial elements may have foci of adenocarcinoma, increasing the risk of malignant degeneration.

The prognosis is however excellent after complete resection.

Hepatic mesenchymal hamartoma (case 2) is a benign hamartomatous growth of mesenchymal tissue in the liver; it occurs in infants and young children less than 2-yearold. It can slowly grow to eventually compress the inferior vena cava or cause respiratory compromise; the $80 \%$ of lesions arise in the right lobe of the liver, and the $20 \%$ are pedunculated.

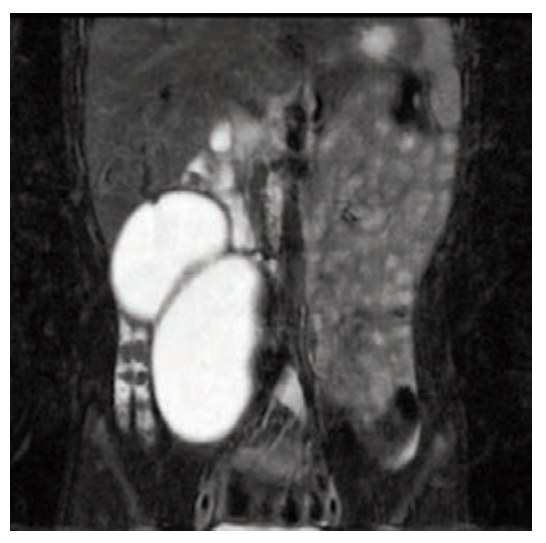

Figure 4. Magnetic resonance imaging coronal view of the cystic mass (retroperitoneal cystic lymphangioma). 
Although cases of spontaneous regression of mesenchymal hamartomas are reported in children, surgical resection is usually the preferred treatment choice, because of a theoretical risk of malignancy and, in symptomatic patients, a mass effect in case of large lesions.

In the differential diagnosis of hepatic cystic lesions the focus should be on the undifferentiated embryonal sarcoma of the liver, a highly malignant tumor of mesenchymal origin, not so rare in children ages 6-10 years, with predisposition to rupture and intratumoral bleeding.

Its treatment strategy include the combination of complete surgical resection and adjuvant chemotherapy.

Mesenteric, omental, and retroperitoneal cysts (case 3,4 and 5) are unusual findings in childhood and many Authors
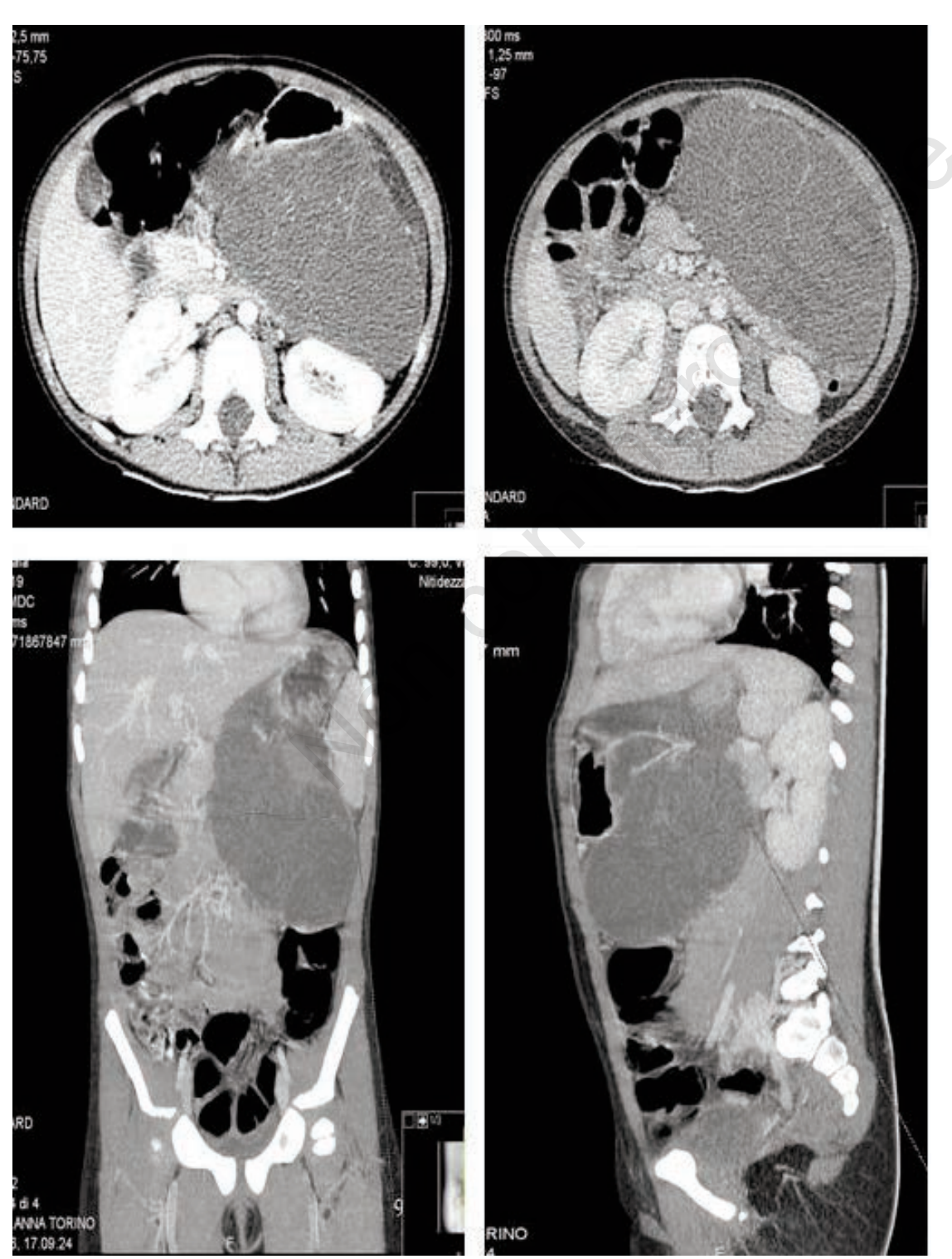

Figure 5. Abdominal Computed tomography scan of a large cystic mass (mesenteric lymphangioma).

suggested that these cysts should be grouped together because of the basis of shared embryologic origin. ${ }^{7}$

Lymphangiomas may occur at all ages with a peak incidence in the third and sixth decades of life, ${ }^{8}$ and $95 \%$ of cases occur in the neck or axilla. 9

The intra-abdominal lymphangiomas in retroperitoneum, omentum, and mesentery are very rare, and account for 3 to $9.2 \%$ of all lymphangiomas, with sites of 59 to $68 \%$ in mesentery, 20 to $27 \%$ in omentum, and 12 to $14 \%$ in retroperitoneum. 10

These cysts could present either incidentally, with nonspecific abdominal complaints or with acute abdominal pain. The spectrum of presentation depends primarily on the location and the size of the cyst.

Any emerging complications, including enlargement, intracystic hemorrhage, tor-

\section{References}

1. Wootton-Gorges SL, Thomas KB, Harned RK, et al. Giant cystic abdominal masses in children. Pediatr Radiol 2005;35:1277-88.

2. Nam SH, Kim DY, Kim SC, Kim IK. The surgical experience for retroperitoneal, mesenteric and omental cyst in children. J Korean Surg Soc 2012;83: 102-6.

3. Esposito C, Alicchio F, Savanelli A, et al. One-trocar ileo-colic resection in a newborn infant with a cystic lymphangioma of the small-bowel mesentery. J Laparoendosc Adv Surg Tech A 2009; 19:447-9.

4. Al-Zaiem MM. Assisted laparoscopic excision of huge abdominal cysts in newborns and infants using the umbilical laparoscopic port incision. J Pediatr Surg 2011;46:1459-63.

5. Gadzijev E, Ferlan-Marolt V, Grkman J. Hepatobiliary cystadenoma and cystadenocarcinoma. Report of five cases. HPB Surg 1996;2:83-92.

6. Senyuz OF, Numan F, Eroglu E, et al. 
Hepatobiliary mucinous cystadenoma in a child. J Pediatr Surg 2004;39:E6-8.

7. De Perrot M, Brundler M, Totsch M, et al. Mesenteric cysts. Toward less confusion? Dig Surg 2000;17:323-8

8. Garcia M, Louis LB 4th, Vernon S. Cystic adrenal lymphangioma. Arch Pathol Lab Med 2004;128:713-4.

9. Kosir MA, Sonnino RE, Gauderer MW. Pediatric abdominal lymphangiomas: a plea for early recognition. J Pediatr Surg 1991;26:1309-13.

10. Okur H, Kucukaydin M, Ozokutan BH, et al. Mesenteric, omental, and retroperitoneal cysts in children. Eur J Surg 1997;163:673-7.

11. Lin JI, Fisher J, Caty MG. Newborn intraabdominal cystic lymphatic malformations. Semin Pediatr Surg 2000;9:141-5.
12. Hancock BJ, St-Vil D, Luks FI, et al. Complications of lymphangiomas in children. J Pediatr Surg 1992;27:220-6.

13. Steyaert H, Guitard J, Moscovici J, et al. Abdominal cystic lymphangioma in children: benign lesions that can have a proliferative course. J Pediatr Surg 1996;31:677-80. 